Document downloaded from:

http://hdl.handle.net/10251/77966

This paper must be cited as:

García Sanjuan, F.; Jaén Martínez, FJ.; Catalá Bolós, A. (2015). Multi-Display Environments to Foster Emotional Intelligence in Hospitalized Children. ACM.

doi:10.1145/2829875.2829880.

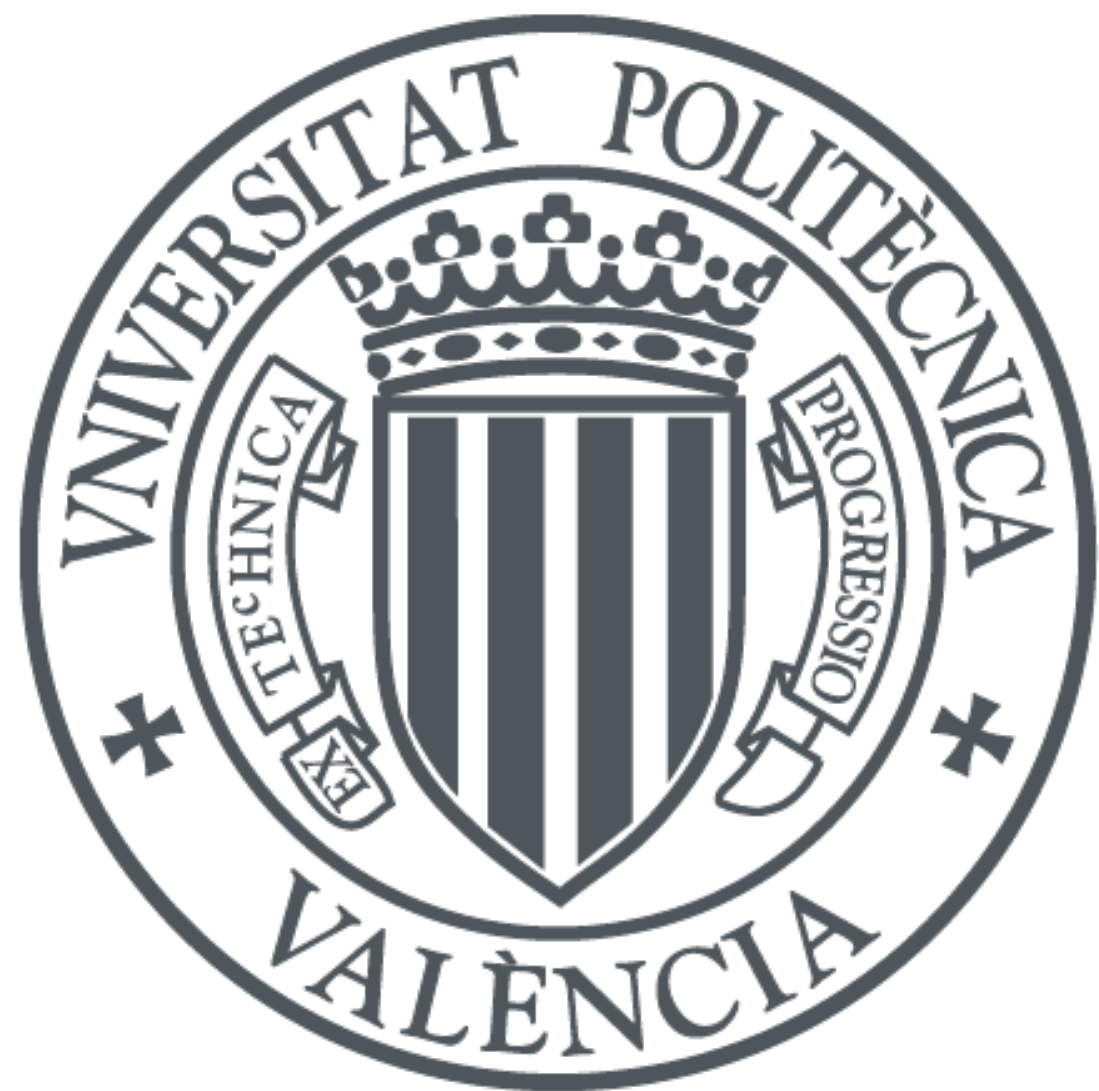

The final publication is available at

http://dx.doi.org/10.1145/2829875.2829880

Copyright ACM

Additional Information

(C) Owner/Author 2015. This is the author's version of the work. It is posted here for your personal use. Not for redistribution. The definitive Version of Record was published in Interacción '15 Proceedings of the XVI International Conference on Human Computer Interactionhttp://dx.doi.org/10.1145/2829875.2829880 


\title{
Multi-Display Environments to Foster Emotional Intelligence in Hospitalized Children
}

\author{
Fernando Garcia-Sanjuan \\ fegarcia@dsic.upv.es
}

\author{
Javier Jaen \\ fjaen@upv.es
}

\author{
Alejandro Catala \\ acatala@dsic.upv.es
}

\author{
DSIC/ISSI, Universitat Politècnica de València \\ Camí de Vera, S/N, 46022 Valencia (Spain)
}

\begin{abstract}
Long-term and frequent hospitalized children are under high loads of emotional stress, which affects their well-being in addition to the illness they are suffering. This thesis proposes and will focus on an approach to use Multi-Display Environments (MDE) in pediatric hospitalization contexts to improve patients' emotional intelligence so they can deal with the negative emotions produced by their situation.
\end{abstract}

\section{Categories and Subject Descriptors}

H.5.2, H.5.3 [Information Interfaces and Presentation (e.g., HCI)]: User Interfaces - Input devices and strategies, interaction styles. Group and Organization Interfaces - Computer-supported cooperative work.

K.3.1 [Computers and Education]: Computer Users in Education - Collaborative learning, Computer-assisted instruction (CAI).

\section{General Terms}

Design, Experimentation, Human Factors, Standardization

\section{Keywords}

Multi-Display Environments (MDE), Hospitalization, Emotional Intelligence, Child-Computer Interaction, Socialization

\section{INTRODUCTION}

According to hospital morbidity statistics from INE for 2013, the number of pediatric hospital stays in 2012 in Spain amounted to $1,813,009$. Besides the fears associated to their illness, frequent and long periods of hospitalization provoke on children high loads of emotional stress and insecurity for being away from their home and their comfort zone with parents and friends. To tackle these problems, there are previous studies that demonstrate that play activities allow children to face the anxiety, conflicts, and fear caused by hospital experiences [4]. However, the application of new technologies in the context of hospital schools has been limited mostly to scenarios of individual use in which emotional stress is addressed in a simplistic way by using technologies to enable communication channels between children and their homes or external schools. However, we not only aim at deviating the children's attention from the clinical processes they are subject to, but also at facilitating the emotions that help them accept their new medical situation and adapt their mental state to face the issues that could arise by means of collaborative game activities.

Since emotional intelligence is mainly of social nature, traditional desktop computers are not the best option to support its training because these devices are mostly designed for individual use. On the other hand, digital tabletops have been shown to be very suitable tools for use in collaborative environments (e.g., [3]). Their multi-touch capabilities allow simultaneous interaction, which contributes to a more democratized participation [9] and a better workspace awareness, since face-to-face settings allow users to know what the others are doing.

Despite research showing these advantages, it is rare to see tabletops embedded in real settings. This is due to a number of disadvantages: their high cost; their limited workspace dimensions, which can only accommodate a certain number of participants; and the fact that their form factor complicates mobility. Therefore, this work will address the development of a collaborative play environment based on multiple interactive surfaces to support the learning of emotional intelligence in pediatric hospitalization contexts aimed at reducing the negative emotional impact caused by long-term or frequent hospital stays.

\section{RELATED WORK}

\subsection{Emotional Intelligence and Tabletops}

Several previous works have considered technology as a way of acquiring emotional intelligence abilities. Morris et al. [6], for example, involve several users collectively around big interactive surfaces in an emotional identification task, through the tagging of different images according to the emotions they cause. During the course of the activity, the researchers observe the users speaking with one another about their feelings towards the images, and, before tagging an image, they self-reflect and ask themselves questions like: “Am I happy?", “Am I sad?" According to the authors, their explorations show people's desire of having technologies that allow the complex expression of emotions through a game experience. However, most of previous works in this area target adults or adolescents, and the activities performed aim mainly at identification of emotions, whereas the practical use of those emotions to solve problems has not yet been fully explored.

Others target children with autistic spectrum disorders (ASD) to increase socialization through technology. As an example, Gal et al. [1] make use of an interactive tabletop to perform storytelling activities to foster collaboration among kids with ASD. They observe an increment of the number of approaches between the children, a higher load of positive affect as well as a higher tendency to express emotions. These type of works, however, are not aimed at making these children reason about their emotions in a way they can overcome an unfavorable personal situation and they rely on interactive tabletops, which may be complex to implant in real hospital environments, as stated previously.

\subsection{Multi-Display Environments (MDE)}

MDE have been object of much previous research. Traditionally, their main purpose was enlarging the visualization space via the physical union of several smaller screens (e.g. [8]), and the interaction played a more secondary role, therefore leading to monouser environments with difficulties to favor collaboration, which is fundamental in emotional learning. Besides, the configurations tended to be static (i.e., the number of screens was fixed a priori), 
and to not allow the mobility of the participants. The posterior popularization of mobile devices, such as phones and PDAs, introduced a higher degree of mobility and multiuser (parallel) interaction since each user could interact with their own device, which they carried with them. Mandryk et al. [5] study the impressions of teenagers when using PDAs in a collaborative game. Because of the reduced dimensions of these devices, the researchers encouraged them to enlarge the visualization space by joining the screens. The participants, however, reported low sense of liberty of movements because they had to maintain the devices together during the course of the activity. Therefore, the mobility was achieved in detriment of the visualization space. This problematic is due to the fact that the approaches commented so far rely basically on joining smaller screens to form a bigger one (and, therefore, rectangular). In this respect, some existing works have proposed alternatives. For instance, Pinch [7] is a technique to couple smartphones and tablets via a manual gesture, and it supports free-form topologies and dynamic modification of the device group to the extent of not needing the displays to be physically joint once coupled. A limitation of this work, however, is that the resulting topology is still flat and the only interaction technique supported is touch. As this analysis suggests, previous research on MDE has mainly focused on technical aspects, and their capabilities have not yet been proven in real settings but rather in experimental contexts in order to show the performance of a given approach. This implicates that, in their design, the ultimate activity to be performed by the users with these MDE has not been considered deeply enough. In our context, the software system and underlying interaction techniques will be considered effective not only if the children are able to perform quickly the manipulations and they understand how to make them, but also if the system supports effectively the construction of experiences that develop emotional intelligence through games.

\section{RESEARCH OBJECTIVES}

From previous works' limitations, we have identified several technical dimensions that will be addressed in our work in order to successfully build collaborative MDE for children in hospitalized environments. These are classified into dimensions regarding the construction of the environment and interaction requirements.

With respect to the former, our approach will allow several displays to form a common environment of any topology, not necessarily the traditional rectangular one, and possibly discontinuous to remove the need for the devices to be physically joint. This way, the users will be able to choose more freely their location. The number of devices will be unbounded, and their annexation to one another will be done in a transparent way in order to avoid complex registration methods. Concretely, our approach to address these requirements will rely on using the built-in camera of tablets and smartphones to track their own position with respect to a wallpaper situated on the ceiling using computer vision algorithms, allowing the system to build a logical map of all the tablets in the group. Since the only requirement is having the wallpaper/marker partially in sight, this approach can also allow some 3D topologies by simply placing the displays in different planes.

Regarding the interactions that our MDE will allow, the most basic one, in order to benefit from the advantages of tabletops in terms of collaboration, is to consider all displays as part of a whole, public surface, and therefore to permit many users interact with the same display at the same time, and also a given user to interact with many devices at once. In order to achieve more dynamic scenarios users will be able to modify the resulting surface by moving the displays through the environment during the course of the collaboration. And, in order to obtain richer ways of interaction, our proposal will support interacting with alternative media such as tangibles, which can result more intuitive for children as they can grab and manipulate actual physical objects. Moreover, it will also accept aerial interactions using gestures with fingers or dedicated objects with the purpose of avoiding interference with another user's actions.

In sum, our goal is to design activities to foster emotional intelligence in hospitalized children. Since this type of intelligence is mainly social, we propose to build collaborative environments using several affordable surfaces such as tablets and smartphones. The dimensions identified previously will then be explored beyond their technical aspects regarding how they impact collaboration among the children and how they foster emotional intelligence that might help them overcome the stressful situations they experience during their hospitalization.

\section{ACKNOWLEDGEMENTS}

This work is supported by Spanish MINECO (TIN2010-20488 and TIN2014-60077-R), from Universitat Politècnica de València (UPV-FE-2014-24), and from GVA (APOSTD/2013/013 and ACIF/2014/214).

\section{REFERENCES}

1. Gal, E., Bauminger, N., Goren-Bar, D., et al. Enhancing Social Communication of Children with High-functioning Autism Through a Co-located Interface. AI \& Society 24, 1 (2009), 75-84.

3. Hornecker, E., Marshall, P., Dalton, N.S., and Rogers, Y. Collaboration and Interference: Awareness with Mice or Touch Input. CSCW'08, ACM (2008), 167-176.

4. Kaminski, M., Pellino, T., and Wish, J. Play and Pets: The Physical and Emotional Impact of Child-Life and Pet Therapy on Hospitalized Children. Children's Health Care 31, 4 (2002), 321-335.

5. Mandryk, R.L., Inkpen, K.M., Bilezikjian, M., Klemmer, S.R., and Landay, J.A. Supporting children's collaboration across handheld computers. CHI EA '01, ACM (2001), 255256.

6. Morris, M.E., Marshall, C.S., Calix, M., Al Haj, M., MacDougall, J.S., and Carmean, D.M. PIXEE: Pictures, Interaction and Emotional Expression. CHI EA '13, ACM (2013), 2277-2286.

7. Ohta, T. and Tanaka, J. Pinch: an interface that relates applications on multiple touch-screen by 'pinching' gesture. $A C E$ '12, Springer-Verlag (2012), 320-335.

8. Ohta, T.Dynamically reconfigurable multi-display environment for CG contents. ACE '08, ACM (2008), 416.

9. Rick, J., Marshall, P., and Yuill, N.Beyond One-size-fits-all: How Interactive Tabletops Support Collaborative Learning. IDC '11, ACM (2011), 109-117. 